\title{
STAZ: Interactive software for undergraduate statistics
}

\author{
VIRGINIA HATCHETTE, ARTHUR R. ZIVIAN, MARILYN T. ZIVIAN, and RONALD OKADA \\ York University, North York, Ontario, Canada
}

\begin{abstract}
STAZ is an interactive computer program that demonstrates statistical concepts, many of which cannot be readily demonstrated using conventional methods. Use of dynamic graphics encourages active engagement with challenging statistical concepts. The program consists of 13 graphical demonstrations, most of which allow for interactive participation by students. A detailed Help file with guided explanations accompanies each demonstration. STAZ is a multiple document interface program that makes full use of Windows features, such as tiling, links, and multitasking. Designed to be used as a supplement for any undergraduate statistics course, STAZ may be used by either instructors in classroom settings or students working independently.
\end{abstract}

Certain concepts in statistics present a challenge to both instructors and students. Many concepts are difficult to express verbally, and most students do not possess sufficient mathematical fluency to understand the meaning of mathematical formulations. When used as an adjunct to conventional methods, dynamic graphics can represent concepts so that they can be grasped by a majority of students, even those with a limited appreciation of sophisticated mathematical formulations.

STAZ is a program that dynamically represents statistical concepts. Incorporating active-learning principles, the demonstrations allow students a hands-on experience with complex statistical concepts. Students control important variables and parameters within demonstrations, and in some cases students act as their own participants in experiments. Concepts like the central limit theorem, effect size, and statistical significance, for example, become more real under the student's control.

\section{OVERVIEW OF STAZ}

The 13 demonstrations are contained within a single program and will operate on any IBM-compatible computer running either Windows 3.1 or Windows 95. STAZ may also be accessed on the Internet. STAZ begins with an introduction for first-time users, but experienced users may go directly to a menu containing a list of the demonstrations: Binomial, Binomial Spinner, Complete Means, Correlation, Regression, Anova, Anova2, Central Limit, Zentral Limit, Student's $t$, Sample Variance, Alpha, and Power.

A menu of detailed Help files can be easily invoked for each demonstration. They are an integral part of the graphic demonstrations and function as tutorials. In Help,

This article was prepared with the financial assistance of the Social Sciences and Humanities Research Council of Canada, through a fellowship to V.H. Correspondence should be addressed to V. Hatchette, Graduate Psychology, York University, 4700 Keele Street, North York, ON M3J1P3, Canada (e-mail: ginna@yorku.ca). students are given general instructions on how to run the demonstration (How do I do it?) and a description of what appears on their screen (What will I see?). Also within Help, the objectives of the demonstration are described (purpose of the demonstration), and students are guided through a series of manipulations (What can I demonstrate?). In a number of demonstrations, students can consult the Teacher's PET (Plain English Translation) for more detailed explanations and concrete examples of the concepts being demonstrated.

\section{DESCRIPTION OF DEMONSTRATIONS}

\section{Binomial and Binomial Spinner}

Binomial is a dynamic, graphic representation of Galton's quincunx. A series of balls descends through eight levels before coming to rest at one of nine compartments. Students manipulate the probability of the balls falling to the left or the right at each of the levels and observe the effect on the distribution of balls. Students see for themselves that the binomial distribution is approximately normal when $p($ left $)=.5$, and skewed (either positively or negatively) when $p$ (left) does not $=.5$. They also observe that the degree of skew is related to the degree of departure from $p($ left $)=.5$. Within Binomial, students are referred to Binomial Spinner for a visual representation that shows how each ball is deflected at every level of the quincunx. After setting the probability that it will point to left or right, students start an arrow spinning within a circle. Each time the arrow comes to rest, a ball travels down one level of the quincunx.

\section{Complete Means}

Complete Means illustrates that the mean of the population and the mean of the distribution of sample means are equal. The population and sample sizes that students can manipulate are kept small so that they can view the complete process of building a distribution of sample means. After the population and sample sizes are selected, 


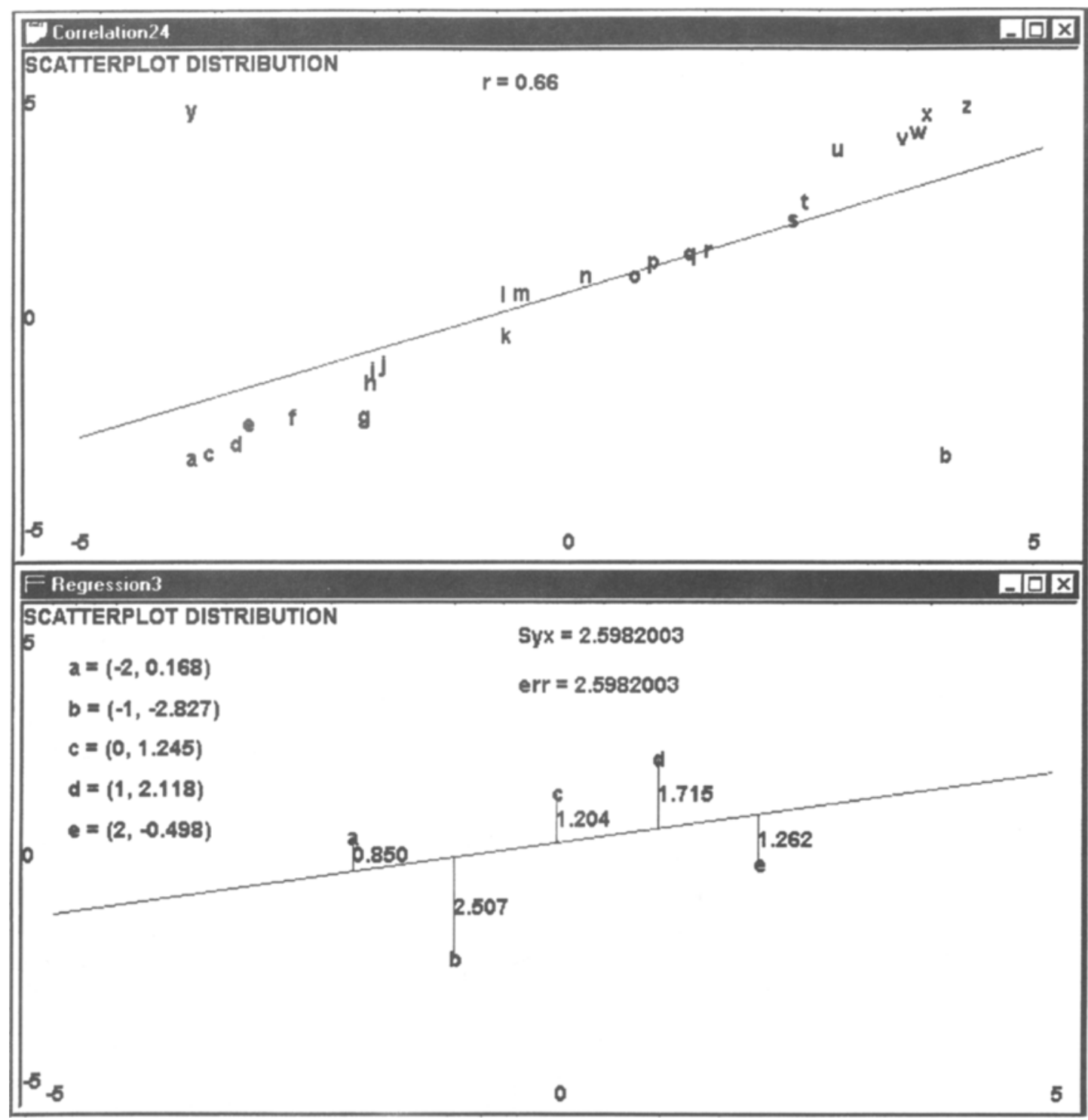

Figure 1. Screen shots of the correlation demonstration after the $y$-coordinates of one pair of data points $(b$ and $y)$ have been interchanged (top half) and of a program-generated regression line (bottom half).

the program displays each of the samples that is drawn. For each sample, a mean is calculated and plotted in the distribution of sample means. When all sample means are plotted, a normal curve is superimposed onto the distribution. The Teacher's PET for this demonstration takes students through a detailed explanation of the relationship between standard deviation and standard error.

\section{Correlation and Regression}

The purpose of Correlation is to give students experience with scatterplots that vary from those that depict a nearly perfect positive correlation to those that depict a nearly zero correlation. The demonstration begins by presenting a scatterplot of data with a correlation approach- ing +1.0 . The value of the correlation is displayed, and a best-fitting regression line is drawn through the scatterplot. Students can change the $y$-coordinates of one pair or a number of pairs of data points and observe the (often dramatic) effect on the value of the correlation and the regression line. For example, the top half of Figure 1 is a screen shot of a scatterplot distribution after the $y$ coordinates of just one pair of data points ( $b$ and $y$ ) have been interchanged.

In Regression, students try to find the best-fitting line for 5 randomly chosen data points. After selecting 2 points with the mouse in order to determine their first guess for the best-fitting line, students can continually adjust the line by moving either its right or left endpoint. With each 


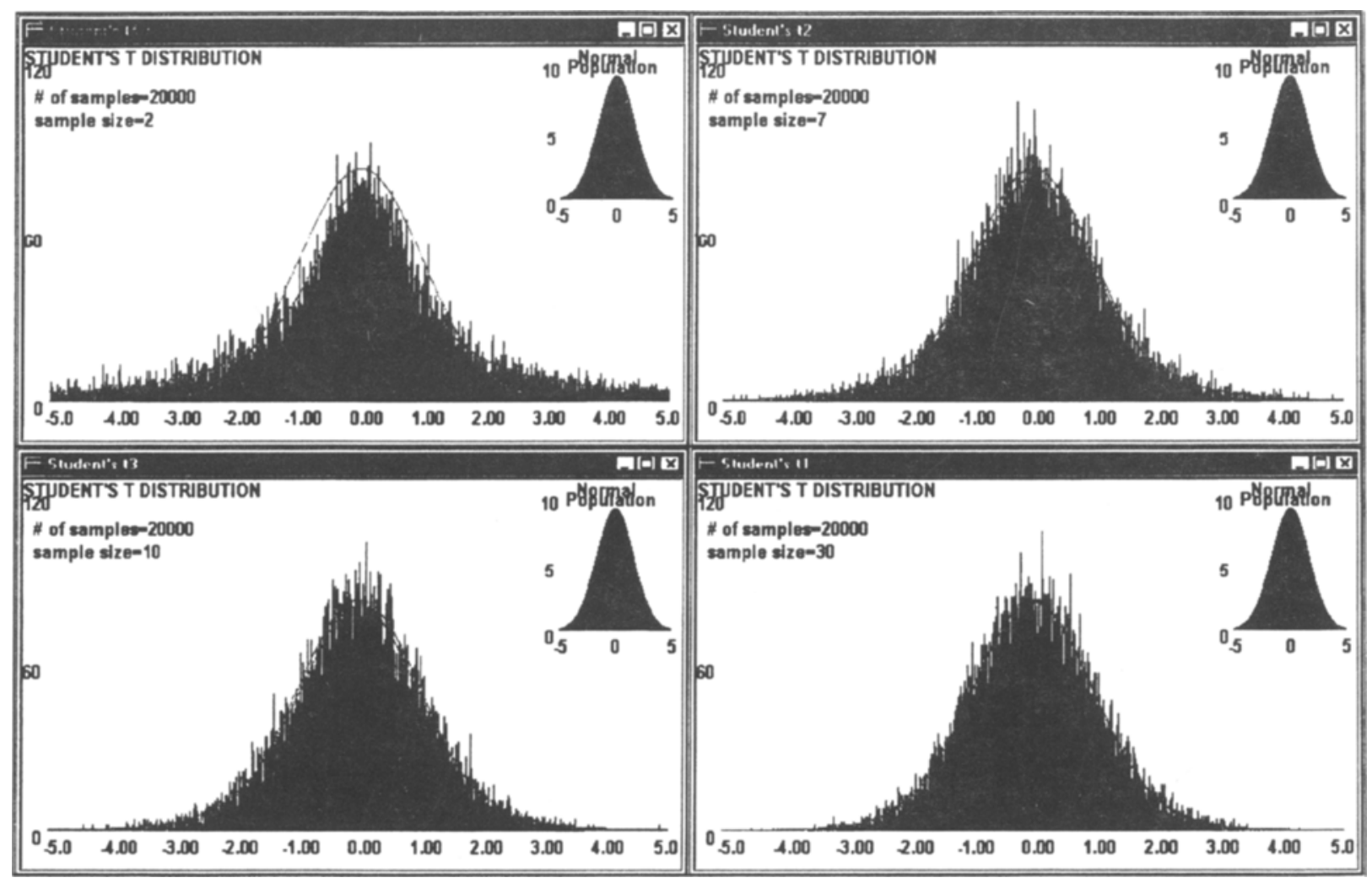

Figure 2. A screen shot of four tiled (sample size $=2,7,10$, and 30) $t$ distributions. (On a color monitor, the $t$ curves are depicted in red, the normal curves in white.)

adjustment the program (1) displays the distance between each data point and the student's line and (2) compares the error in prediction given by the student's line to the least squared error. At any time, students can ask the program to draw the best-fitting (regression) line. With repeated attempts to decrease their errors in prediction, students gain experience in visually judging the regression line and increase their appreciation of what is meant by the bestfitting line. A screen shot of the regression line generated by the program is displayed in the bottom half of Figure 1.

\section{Anova and Anova2}

Students act as their own participants in either a onefactor (Anova) or two-factor (Anova2) experiment involving analysis of variance (ANOVA). In Anova, students are confronted with 16 moving targets ( 8 fast and 8 slow) consisting of three embedded squares. Targets are presented one at a time, and student players try to "hit" the target with their mouse-controlled cursor before the target disappears at the right side of the screen. Scores depend on how close to the center each of the targets is "hit."

In Anova2, students are presented with 32 moving targets ( 16 fast and 16 slow). Half of the targets travel across the entire screen from left to right; the remaining targets disappear after traveling across the first quarter of the screen. When the Anova and Anova2 experiments are finished, students calculate $F$ statistics on their data to see whether their performance differed as a function of target speed (Anova) or screen distance, and/or their interaction (Anova2). In both demonstrations, students are warned that the generated data are analyzed with an $F$ test for illustration purposes only and that repeated measures single-subject data violate the assumption of independence for the common ANOVA $F$ test.

\section{Central Limit, Zentral Limit, Student's $t$, and Sample Variance}

These four demonstrations share many common features. Students select the number of samples to be drawn $(1,000-50,000)$, the sample size $(1-100)$, and the population (triangular, uniform, V-shaped, or normal) from which the samples are to be drawn. The student controls the speed of the demonstration, which can range from one value plotted every $8 \mathrm{sec}$ to the entire distribution being plotted in approximately $3 \mathrm{sec}$. Students can also pause the demonstration at any time. To facilitate comparisons of distributions obtained from different populations and sample sizes, students may "tile" up to 25 windows.

In Central Limit, students observe the effect of number of samples and sample size on the distribution of sample 


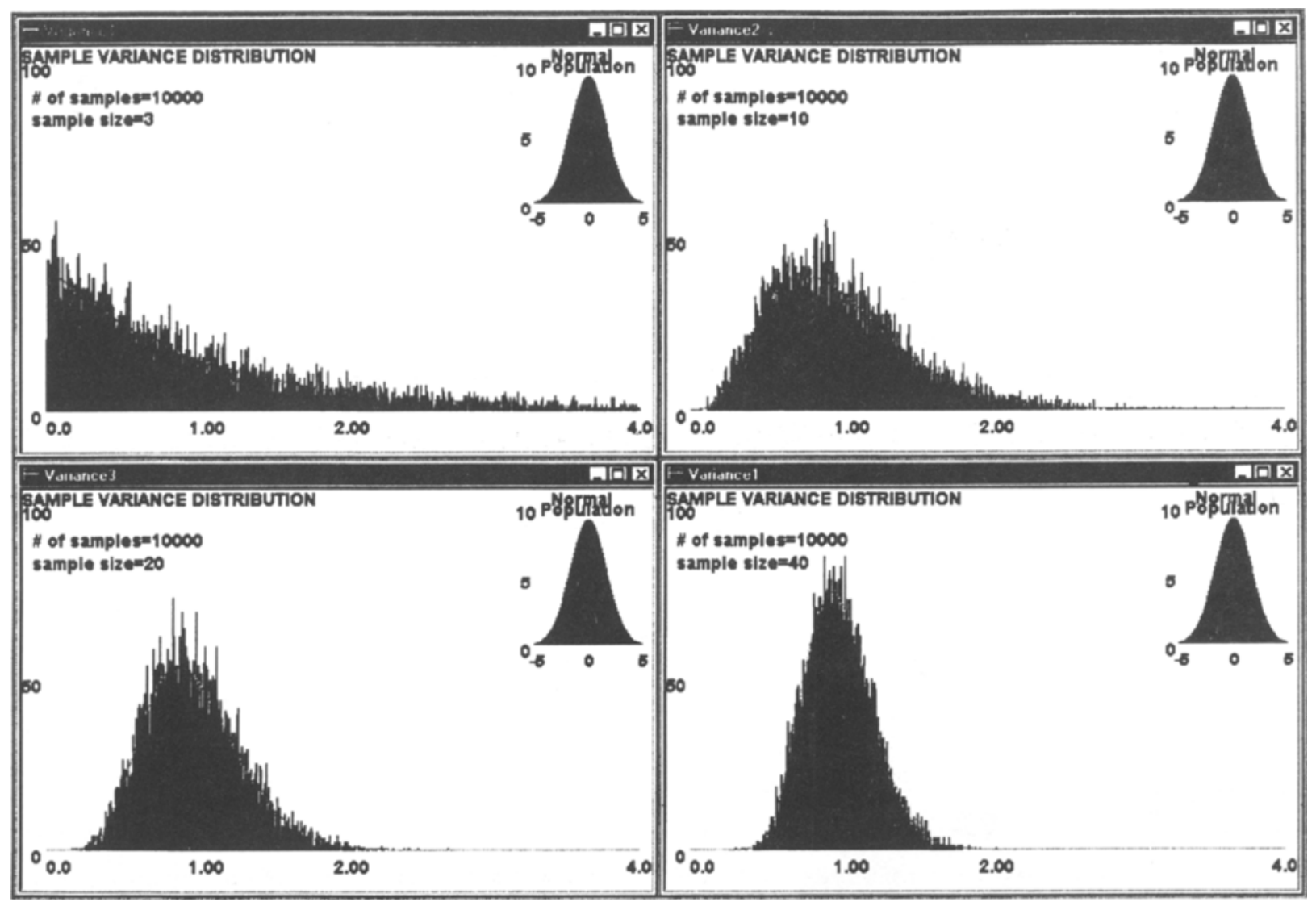

Figure 3. A screen shot of four tiled sample variance distributions of increasing sample size $(3,10,20$, and 40$)$.

means. The program displays the sample values, calculates the mean for each sample, and plots these means in a distribution of sample means. When these operations are complete, a normal curve is superimposed onto the distribution of sample means. The Help file contains guidelines for varying the population, the number of samples, and the sample size so that their influence on the shape and standard error of the distribution of sample means can be observed. The Teacher's PET for Central Limit introduces Professor D. Mented, who uses an unusual grading scheme incorporating a series of 10 "pop" quizzes. Professor D. Mented has been teaching introductory statistics for 45 years and has saved the marks from all of her students' "pop" quizzes. The Teacher's PET demonstrates how these marks may be used to construct a distribution of sample means.

Zentral Limit illustrates that (1) the mean is always equal to zero and (2) the standard deviation will always be equal to one. Student's $t$ illustrates that (1) the distribution of Student's $t$ approaches a normal shape when scores are drawn from a normal population, and (2) there is a family of Student's $t$ distributions. Sample values are displayed, the mean and $z$ score (or $t$ score) for each sam- ple are calculated, and the $z$ score (or $t$ score) is plotted in a distribution. When these operations are complete, a normal curve is superimposed onto the distribution of $z$ scores. Both a normal and a $t$ curve are superimposed onto the distribution of $t$ scores. Students are encouraged to vary the population and the sample sizes in order to observe how they influence the shape of the distributions. Figure 2 displays a screen shot of four (sample size $=2$, 7,10 , and 30) $t$ distributions that have been tiled. On a color monitor, the $t$ curves are depicted in red, the normal curves in white, and students can observe how the distribution of $t$ scores comes closer and closer to approximating a normal distribution as sample size increases. Professor D. Mented appears again in the Teacher's PET for Student's $t$. This time, however, nothing is known about how the population of "pop" quiz scores is distributed other than that it has a mean $(\mu)$ of zero.

The purpose of Sample Variance is to demonstrate that, for normal populations, distributions of sample variance are positively skewed, especially for small sample sizes. The program displays the sample values, calculates the mean and variance for each sample, and plots each variance in a distribution of sample variance. When these op- 
erations are complete, a chi-square curve is superimposed onto the distribution. Students are able to vary the population and the sample size to observe their influence on the shape of the distribution of sample variance. Figure 3, a screen shot of four sample variance distributions of increasing sample size $(3,10,20$, and 40$)$, displays the dramatic differences in the distribution of sample variance that can be obtained for samples of varying sizes.

\footnotetext{
Alpha and Power

Alpha and Power are the least interactive of the demonstrations. Alpha graphically depicts alpha levels and Type I error. Students select a one- or two-tailed Student's $t$ or $z$ distribution, or a chi-square distribution. The distribution is displayed with the critical region shaded. By following concrete examples in the Help file and by using this demonstration in conjunction with either Zentral Limit or Student's $t$, students are helped to appreciate the relationship between alpha and Type I error.

Three distributions, one representing the null hypothesis and two representing alternate hypotheses, are displayed in Power. By holding variance, effect size, and alpha constant, students can manipulate sample size and observe its effect on the power of a one-tailed $z$ test by observing changes in the shaded areas in the distributions that represent the alternate hypotheses.
}

\section{USING STAZ}

Since STAZ is designed as an adjunct to basic statistics courses and texts, there is a great deal of flexibility in how the program may be used. Instructors and/or students may move freely from one component of the program to any other. Instructors may use STAZ with an overhead PC projection device as they lecture or as part of a statistics laboratory. Students may use STAZ to re-create and review lecture and laboratory demonstrations. Furthermore, because STAZ is entirely self-contained and user friendly, students may use it independently as a study aid.

STAZ may be obtained by ordering diskettes from V.H. for $\$ 5$ to cover disk and mailing costs; or, either of the compressed (by PKZIP 2.0) files STAZ31.ZIP or STAZ95.ZIP may be downloaded from http://www. yorku.ca/faculty/academic/mzivian/staz95.htm or http:// www.yorku.ca/faculty/academic/mzivian/staz31.htm, respectively.

A slower version of STAZ may be viewed (with a Java-enabled browser) at our website: http://www. yorku.ca/faculty/academic/mzivian.

(Manuscript received October 21, 1997; revision accepted for publication June 23, 1998.) 